\title{
DIFFERENTIAL DIAGNOSIS OF PSORIASIS
}

\section{P. LISI}

Section of Clinical, Allergological and Venereological Dermatology, Department of Medical and Surgical Specialities and Public Health, University of Perugia, Perugia, Italy

\begin{abstract}
SUMMARY
The clinical diagnosis of psoriasis is relatively easy, especially when the lesions consist of erythematous, silvery white scaly, sharply demarcated, indurated plaques, distributed symmetrically on the extensor surfaces of limbs, the lower back and the scalp. These clinical features reflect the histopathological findings observed in active lesions, characterized by parakeratosis, acanthosis of the epidermal ridges, tortuous and dilated blood vessels, and perivascular leukocytic infiltrate; the Munro microabscess and the spongiform pustule of Kogoj are diagnostic. Diagnostic doubts, however, may arise in several clinical variants and atypical cases (guttate psoriasis, follicular or spinulosic psoriasis, erythrodermic psoriasis, pustular psoriasis) or when the psoriatic lesions are localized in particular sites (palms, soles, scalp, body folds, penis, nails). The value of erythemato-papulosquamous psoriasiform eruptions occurring during or after the administration of a diagnostic or therapeutic agent especially in psoriatic subjects is discussed.
\end{abstract}

Key words: Psoriasis, differential diagnosis, skin diseases, clinical features, histopathological aspects

\section{INTRODUCTION}

The clinical diagnosis of psoriasis $(\mathrm{P})$ is relatively easy, especially when the lesions consist of erythematous ("salmon pink"), silvery white scaly, sharply demarcated, indurated plaques. They have an oval or irregular shape, vary from one to several centimetres in diameter and are distributed symmetrically on the extensor surfaces of limbs (mainly elbows and knees), the lower back and the scalp. Itching is very variable, but it is usually absent. The degree of itching is often conditioned by the emotional state of the patient and, if severe, may be a symptom of anxiety or depression (1). These clinical features reflect the histopathological findings observed in active lesions, characterized by hyperkeratosis, parakeratosis, diminution or loss of the granular cell layer, acanthosis of the epidermal ridges, tortuous and dilated blood vessels, and perivascular leukocytic infiltrate in the dermal papillae. Typically, the epidermal ridges are evenly elongated and club-shaped at the tips. Two other changes are diagnostic: the Munro microabscess

Corresponding author:

Paolo Lisi, MD

Dermatologia Clinica, Allergologica e Venereologica

Policlinico - 06122 Perugia, Italy

E-mail: plisi@unipg.it and the spongiform pustule of Kogoj, due to the small accumulation of neutrophils, respectively within the parakeratotic stratum corneum and the spongiotic epidermis.

\section{DIFFERENTIAL DIAGNOSIS}

The clinical and histological aspects of chronic $\mathrm{P}$ vulgaris are generally sufficient to make the diagnosis. Diagnostic doubts, however, may arise in several clinical variants and atypical cases or when the psoriatic lesions are localized in particular sites, especially in patients with type II P. In these patients the cutaneous manifestations appear in their 50s (2) and, unlike type I, do not show familial segregation and are much less significantly associated with specific HLA phenotypes (Cw6, B13, B17, DR7) (3). The most common onset of type I $\mathrm{P}$ is in young adults.

\section{Clinical variants and atypical cases}

Guttate psoriasis is the most frequent $\mathrm{P}$ variant observed in children and young adults. It is characterized by an acute eruption of numerous, small (0.3-1.0 cm diameter), round or slightly oval, erythematous and scaly papules and plaques which are widely disseminated, particularly on the trunk and proximal part of the extremities. The face could 
also be involved. The lesions typically appear one or two weeks after a severe streptococcal infection of the upper respiratory tract.

The principal differential diagnosis includes some papulosquamous or erythematosquamous disorders, such as pityriasis lichenoides chronica, secondary syphilis, lymphomatoid papulosis and pityriasis rosea. In the first, the recurrent lesions are usually less evenly scattered, have a brownish red or orange-brown colour, are capped by a single detachable opaque mica-like scale, and often leave hypopigmented macules. Papules of pityriasis lichenoides chronica as those of guttate $\mathrm{P}$ are asymptomatic, are localized on the trunk and upper limbs and appear prevalently after a pharyngotonsillar infection in pediatric population.

The non-pruriginous papulosquamous lesions of late secondary syphilis may mimic a guttate $\mathrm{P}$, but the coppery red colour and the collarette of scales of papules, the presence of other cutaneous signs of papular syphilis (e.g. condylomata lata, P-like plaques of the palms and soles, corona veneris) and the generalized lymphadenopathy with enlarged indolent lymph nodes remove any diagnostic doubts even before the results of serological testing are known.

Lymphomatoid papulosis, which sometimes may be confused with guttate $\mathrm{P}$, is a chronic, recurrent, self-healing papulonecrotic or papulonodular skin disease with histologic features suggestive of a malignat lymphoma (CD30-positive). The coexistence of red-brown papules, nodules with ulcerated necrotic centres, hypopigmented or hyperpigmented macules and, occasionally, fine atrophic circular or varioliform scars predominantly affecting the trunk and limbs is diagnostic.

Pityriasis rosea is a common acute self-limited papulosquamous eruption, probably viral, which is more frequent in adolescents and young adults. The classical lesions are pink or salmon in colour, oval in shape and with a long axis oriented along the Langer's cleavage lines; they are usually distributed on the trunk and proximal extremities. These lesions are easily distinguishable from those of guttate $\mathrm{P}$ because of their appearance; they are larger in size, with small fine scales, squamous marginal collarette and a "fir-tree" or "Christmas tree" distribution on the posterior trunk. The onset of a solitary herald patch which is wider than the lesions of the later eruption and the spontaneous resolution in 6-8 weeks are pathognomonic for pityriasis rosea. The erythemato-papulosquamous psoriasiform eruptions occurring during or after the administra- tion of a diagnostic or therapeutic agent may mimic an eruptive $\mathrm{P}$, especially in patients with $\mathrm{P}$ or a psoriatic diathesis. In these cases the bright red colour of the lesions, less abundant scaling, more severe itching, eosinophilia and the variable number of eosinophils in the dermal infiltrate are more typical of a drug reaction. However, the diagnosis is often retrospective. Several drugs are known to exacerbate a pre-existing $\mathrm{P}$ (antimalarials and, sometimes, non-steroidal anti-inflammatory drugs, angiotensin-converting enzyme inhibitors) or trigger off the onset of P lesions in normal and psoriatic subjects (lithium salts, beta-blocking agents, interferon-alfa) after a variable latent period.

Follicular or spinulosic psoriasis is rare but more frequent in children. It may be mistaken for pityriasis rubra pilaris which is characterized by rough follicular papules on an erythematous base (dark red), especially on the dorsal aspect of the fingers, and by large orange-red plaques with distinctive "islands of sparing". An orange-red waxy palmar and plantar keratoderma is also peculiar. Histologically pityriasis rubra pilaris differs from $\mathrm{P}$ due to the presence of alternating parakeratosis and hyperkeratosis in both vertical and horizontal directions (spotty parakeratosis). Neutrophil infiltration of the stratum corneum is not a feature of pityriasis rubra pilaris unless there is a secondary infection (4).

Erythrodermic psoriasis is a generalized redness and scaling, often associated with oedema, which involves more than $90 \%$ of the skin surface. Its onset can be precipitated by the rapid withdrawal of systemic or topical corticosteroid after prolonged use, treatment with antimalarials, acute infections, hypocalcaemia, over-exposure to sunlight or UV irradiation when the disease is in a Koebner-positive phase. The differential diagnosis with other erytrodermas (Tab. I) is not always easy, but previous plaques in classic locations, nail involvement, facial sparing, inflammatory arthritis and family history of $\mathrm{P}$ are diagnostic clues. Itching may be severe, but it is more typical and severe in erythrodermas due to eczematous dermatitis or Sèzary syndrome, in which lymphadenopathies are also a more common finding. In spite of multiple skin biopsies and clinical investigations, the underlying cause of erythroderma is not found in about onethird of patients (5).

Pustular psoriasis (PP) (generalized PP or von Zumbusch pattern, generalized PP of pregnancy or impetigo herpetiformis, localized PP on palms and soles or Barber pattern) and its variants (circinate 
Table I - The main underlying causes of erithrodermas in neonates, infants and adults (from Sterry and Muche (5), modified).

\begin{tabular}{|lll|}
\hline Common causes & Less common causes & Rare causes \\
\hline Atopic dermatitis (also in infants) & Contact dermatitis & Ofuji's papulo erithroderma \\
Psoriasis (also in infants) & Seborrheic dermatitis & Autoimmune connective tissue disorders \\
Drug reactions & Chronic actinic dermatitis & (dermatomiositis, subacute lupus erythematosus) \\
Cutaneous T-cell lymphoma & Pityriasis rubra pilaris & Norwegian scabies \\
(Sèzary syndrome, mycosis fungoides) & Bullous dermatoses (pemphigus foliaceus, & Hypereosinophilia syndrome \\
Congenital ichthyoses (bullous and not) & bullous pemphigoid, paraneoplastic pemphigus) & Lichen planus \\
(in neonates and infants) & Netherton syndrome (in infants) & Dermatophyte infection (also in infants) \\
& Staphylococcal scalded skin syndrome (in neonates) & Wiskott-Aldrich syndrome (in infants) \\
& Omenn's syndrome (in neonates) & Graft-versus-host disease (in infants) \\
\hline
\end{tabular}

Table II - Histological classification of amicrobic pustular eruptions (6).

\begin{tabular}{|ll|}
\hline Spongiform or multilocular intraepidermal pustules & Non-spongiform or unilocular intraepidermal pustules \\
\hline Pustular psoriasis & Subcorneal pustular dermatosis or Sneddon-Wilkinson disease \\
Post-streptococcal acute generalized pustolosis & IgA intraepidermal neutrophilic dermatosis Infantile acropustolosis \\
Acute generalized exanthematous pustolosis Fiessinger-Leroy-Reiter syndrome & Pustular vasculitis of the hands \\
Generalized pustolosis associated with hypocalcaemia caused & \\
by post-surgical hypo-parathyroidism, chronic renal failure, .. & \\
\hline
\end{tabular}

or Bloch-Lapière's pattern, exanthematic, acrodermatitis continua of Hallopeau) are characterized by non-follicular sterile pustules, which represent the macroscopic aspect of the massive neutrophil infiltration of epidermis. Focal infections, corticosteroid therapy, topical irritants, pregnancy, hypocalcaemia and stress have been reported as triggering factors. Smoking may aggravate the course of lesions localized on the palmoplantar surfaces.

All of these variants must be distinguished from other amicrobic pustular eruptions, in which the histological aspect of the lesions is sometimes spongiform or multilocular as in pustular $\mathrm{P}$, but more often it is non-spongiform or unilocular (Tab. II). The most important diagnostic problems are raised by acute generalized exanthematous pustolosis, particularly in patients with a family and/or personal history positive for $\mathrm{P}$, and subcorneal PP.

Acute generalized exanthematous pustolosis (AGEP) is an acute febrile drug eruption characterized by numerous small, primarily non-follicular sterile pustules, arising within large areas of edematous erythema, which also happens in generalized pustular psoriasis.

The acuteness of lesions, their first appearance on the cutaneous folds and face, the following rapid dissemination, the frequent association with other skin manifestations (purpura, vesicles, erythema multiforme like lesions), the intense itching, the high fever, and the relatively short latent time between the drug administration and the skin eruption (1-2 days) make the diagnosis of AGEP possible. Neutrophilic leucocitosis and the mucous involvement, on the contrary, are not diriment because they are present in both diseases; in particular, the circinate scaling of the tongue is a typical sign of PP (benign migratory glossitis). Histological features can also be helpful. Massive edema and mixed perivascular inflammatory infiltrate in the superficial dermis, exocitosis of eosinophils and necrosis of several keratinocytes are more suggestive of AGEP, while parakeratosis and papilloacanthosis are more characteristic of PP.

Subcorneal pustular dermatosis (Sneddon-Wilkinson disease) may be easily differentiated from PP, whether clinically or histologically. The annular, polycyclic or serpiginous lesions with a scaly edge, the relapsing flaccid vesicopustules with clear fluid in the upper half and pus in the lower half, and the absence of spongiform changes are diagnostic, as is the positive response to dapsone. In other cases, mainly when the pustules are asymmetrically localized on the palmar and plantar regions, infective conditions (e.g. pustular dermatophytoses, bacterial impetigo) and eczematous dermatitis (e.g. dyshidrosis, irritant or allergic contact dermatitis) must be excluded by carrying out PAS or GRAM stains and cultures of biological material, and patch test. 


\section{Morphological modifications by lesion localization}

Psoriatic lesions exhibit a considerable degree of morphological uniformity, even if they might show regional variations. Those observed on palms and soles are the most significant, probably because of the skin thickness and the trauma related to occupational activities and/or hobbies. The sharply defined edge of plaques, the orange-red colour, the absence of vesiculation and the dull red thickening of the skin on the knuckles are helpful to exclude keratotic eczema, lichen simplex or secondary syphilis. Nail changes are diagnostic. Palmo-plantar P, nevertheless, may be eczematous, mainly in children and in those with a positive family history for $\mathrm{P}$ and/or atopy, or lichenoid. The violaceous colour, glistering surface and oral changes are suggestive of lichen planus.

The scalp is one of the most common sites for P. Its involvement may be diffuse or in multiple discrete plaques of varying size. In contrast to seborrhoeic dermatitis, psoriatic patches are well-defined, silvery white, xerotic, pruriginous, and often advance beyond the hairline. Sometimes, the scales have an asbestos-like appearance and are firmly attached to the scalp hairs (pityriasis amiantacea). However, the differential diagnosis may be difficult, especially in subjects without psoriatic lesions on classic sites. In these cases also histological aspects are not decisive, although the spongiotic component is less cospicuous or absent in $\mathrm{P}$.

Flexural or inverse $\mathrm{P}$ is characterized by the development of shiny, pink to red, sharply demarcated thin plaques in the body folds (axillae, submammary creases, inguinal folds, intergluteal cleft, retroauricolar folds), which are commonly fissured at the depth. As this $\mathrm{P}$ pattern is one of the causes of intertrigo, it must be differentiated from other etiologies as bacterial intertrigo, Candida intertrigo, seborrhoeic dermatitis and, less often, erythrasma, contact dermatitis, Hailey-Hailey disease, flexural Darier's disease (Table III). The potassium hydroxide examination and the culture of scales, Wood's lamp examination may be useful to exclude fungal and/or bacterial infections.

Scaling is also greatly reduced or absent in $\mathrm{P}$ of the penis, but the colour of patches and their well-defined edges are usually distinctive. In some cases, especially when only a lesion is present, a diagnostic biopsy is necessary to exclude Zoon's plasma cell balanitis, erythroplasia, Bowen's disease or extramammary Paget.

Toenails and mainly fingernails are frequently involved in all types of $\mathrm{P}$, especially in psoriatic arthropathy. Pitting, oil spots, subungual hyperkeratosis and onycholysis are the most common but are not patognomonic disorders. However, pits or punctate depressions of the nail plate due to a defect of the nail matrix are deeper in $\mathrm{P}$ than in alopecia areata, contact dermatitis or occupational trauma. Also oil spots, caused by a separation between the nail plate and nail bed due to the accumulation of large serum-like exudate containing glycoproteins, may be found in inflammatory and eczematous diseases. They have been reported in systemic lupus erythematosus (7). Subungual hyperkeratosis is very frequent in $\mathrm{P}$ and results from repeated trauma and numerous other chronic inflammatory conditions involving the nail bed or hyponychium, such as onychomycosis, contact dermatitis and

Tabella III - The most common causes of intertrigo and their clinical features.

\begin{tabular}{|ll|}
\hline Skin disorders & Clinical features \\
\hline $\begin{array}{l}\text { Infections: } \\
\text { bacterial intertrigo } \\
\text { erythrasma } \\
\text { Candida intertrigo }\end{array}$ & $\begin{array}{l}\text { Eczematoid dermatitis, fissuring in the depth of affected flexure } \\
\text { Red-brown patches, furfuraceous scales in the margins } \\
\text { Clistening deep red colour and little moist exudation of the fold, scaling confined to the edge, } \\
\text { small satellite pustules and tiny erosions outside the main area, soreness and itching }\end{array}$ \\
\hline $\begin{array}{l}\text { Eczematous dermatitis: } \\
\text { contact dermatitis } \\
\text { atopic dermatitis }\end{array}$ & $\begin{array}{l}\text { Reddening, erosions, exudation, maceration, crusts, burning } \\
\text { Poorly demarcated erythema, papules, crusts, lichenification, itching }\end{array}$ \\
\hline $\begin{array}{l}\text { Miscellanea: } \\
\text { psoriasis } \\
\text { seborrhoeic dermatitis } \\
\text { Hailey-Hailey disease } \\
\text { flexural Darier's disease }\end{array}$ & $\begin{array}{l}\text { Shiny, pink to red, sharply demarcated thin plaques, itching } \\
\text { Yellowish-red lesions, bran-like scaling }\end{array}$ \\
\hline
\end{tabular}


pityriasis rubra pilaris. Finally, onycholysis is the most aspecific nail alteration in psoriatic patients because the detachment of the nail from the bed is due to many systemic, congenital, cutaneous, physical, chemical and infective causes.

\section{REFERENCES}

1. Griffiths CEM, Camp RDR, Barker JNWN. Psoriasis. In: Burns T, Breathnach S, Cox N, Griffiths C. Rook's textbook of dermatology. $7^{\text {th }}$ edition. Blackwell: Malden, 2004; 35.1.

2. Henseler T, Christophers E. Psoriasis of early and late onset: characterization of two types of psoriasis vulgaris. J Am Acad Dermatol 1985; 13: 450-456.
3. Henseler T. The genetics of psoriasis. J Am Acad Dermatol 1997; 37: S1-S11.

4. McKee PH, Calonje E, Granter SR. Pathology of the skin. $3^{\text {rd }}$ edition. Elservier Mosby: Philadelphia, 2005; 195-206.

5. Sterry W, Muche JM. Erythroderma. In: Bolognia JL, Jorizzo JL, Rapini RP. Dermatology. Mosby: London, 2003;165-174.

6. Saurat J-H, Borradori L. Pustolosi amicrobiche. In: Saurat IH, Grosshans E, Laugier P, Lachapelle J-M. Dermatologia e malattie sessualmente trasmesse. $3^{\mathrm{a}}$ edizione. Masson: Milano, 2006; 591-603.

7. Baran R, Dawber RPR, Haneke E, Tosti A. Nail plate and soft tissue abnormalities. In: Baran R, Dawber RP, Haneke E, Tosti A, Bristow I. A text atlas of nail disorders: techniques in investigation and diagnosis. $3^{\text {rd }}$ edition. Martin Dunitz: London, 2003; 63-80. 